ALPHA N 26 Julio / 2008 (217-232)

ISSN 0716-4254

http://alpha.ulagos.cl

\title{
UNA DISCUSIÓN EN TORNO A LA VERDAD EN CIENCIAS Y HUMANIDADES
}

A discussion around the truth in sciences and humanities ${ }^{1}$

Jorge Gibert Galassi*

\section{INTRODUCCIÓN}

El propósito del trabajo es discutir el estado de la verdad, así como algunas de sus propiedades y características, en ciencias y humanidades. Para ello, trato de mostrar todas las doctrinas más importantes de la verdad, de modo breve pero concluyente, tanto desde las perspectivas científicas como filosóficas. Se ilustra el vínculo entre la verdad, los hechos y las creencias; así como las posibilidades de aplicar ciertas doctrinas de la verdad en disciplinas específicas. En virtud del carácter de sus objetos de estudio, la hipótesis subyacente es que el tratamiento de la verdad difiere de una disciplina a otra.

En una de sus muchas conversaciones radiales, el poeta y periodista Osvaldo Ferrari le dice a Jorge Luis Borges que la fe y la falta de fe podrían ser dos formas de aproximarse a la verdad, a lo que Borges le contesta: "Yo creo haber sido un hombre de fe". Extraña afirmación, siendo Borges no creyente. Pero agregó: "Tengo fe en la ética, y tengo fe en la imaginación". Probablemente Borges sugirió que las creencias éticas y especulativas juegan un rol en la búsqueda de la verdad.

Una de las creencias contemporáneas señeras, especialmente durante el siglo XIX, ha sido identificar la verdad con la ciencia. El entusiasmo por las ininterrumpidas confirmaciones de la mecánica clásica de Newton insufló de optimismo a todo el mundo. Contagió también a las ciencias sociales, cuando Comte definió la sociología como física social. Se llegó a pensar que el conocimiento absoluto sólo era una cuestión de tiempo. La llegada del siglo $\mathrm{XX}$ trajo consigo a la díscola mecánica cuántica, que con la arremetida del probabilismo de Bohr, el principio de no localidad, etc. sepultó tales esperanzas. Pero el descalabro no sólo provino del mundo de la ciencia experimental. A mediados del siglo pasado el filósofo británico Alfred Ayer y

${ }^{1}$ Este trabajo forma parte del proyecto FONDECYT No. 11060031 (2006-2008). El autor agradece el apoyo de CONICYT-Chile. 


\section{Jorge Gibert Galassi}

el norteamericano Roderick Chisholm, postularon una definición del conocimiento proposicional ("S sabe que p"), cuya consecuencia es la hoy denominada definición tripartita del conocimiento, esto es, que el conocimiento es la creencia verdadera justificada. Según esta definición, creencia, verdad y justificación son las condiciones individualmente necesarias y conjuntamente suficientes del conocimiento: no puede haber conocimiento si falta alguna de ellas, como no puede ser el caso que se den las tres y no lo haya. En 1963, Edmund Gettier publicó un breve artículo en el que ofrecía dos contraejemplos a la definición tripartita del conocimiento. La discusión de estos contraejemplos constituye lo que se conoce como el "Problema de Gettier", hoy sin solución. Para rematar, Thomas Kuhn — según él, sin esa intención (1992) - escribió en 1962 La estructura de las revoluciones científicas, donde la noción de paradigma reemplazó en la práctica la visión unificada y progresiva del conocimiento y ayudó a masificar la idea de que la verdad científica es relativa. La doctrina actual podría resumirse diciendo que la verdad, como algo válido para cualquier persona, ha sido reemplazada por la verdad "para algunos" o "para alguien", lo que cuestiona la misma esencia de nuestra concepción intuitiva de la verdad.

Aparentemente, la verdad ya no es identificable con la ciencia y ni siquiera es una característica primordial de ella o de las humanidades. ¿Cómo es posible? ¿Habrá algún error en el planteamiento?

Explicitando mis propias creencias, adhiero a la idea que la ciencia aspira a la verdad sustantiva sobre el mundo, en una $u$ otra forma. Sin esa aspiración la ciencia no tendría sentido, aunque sabemos por la historia, que los científicos se han equivocado anteriormente, en especial, postulando teorías en que las representaciones de sus objetos eran muy parciales o equivocadas. Esto ha sido frecuente en física (heliocentrismo, teoría de la gravedad, teoría general de la relatividad). Pero, por lo mismo, la aproximación "mejorada" a la verdad es históricamente posible. Se sabe, también, que los científicos no tienen la gloria de Beatriz, capaz de bajar a los infiernos para salvar a Dante, y que, como humanos que son, en reiteradas ocasiones han saboteado la verdad y la han acomodado a sus intereses. Basta recordar la Alemania Nazi o la Ex URSS. Independientemente de las disciplinas, es común que lo falso o equívoco se filtre en la actividad científica, como lo sugieren Michael Foucault (1999) y Pierre Bourdieu (2003). Sin embargo, la ciencia siempre ha trabajado por la verdad, aun en el intento de van Fraassen, que planteó al menos "salvar el fenómeno" como representación y consideró la ciencia como un empirismo constructivo (1980).

También adhiero a la idea de que la ciencia siempre ha concebido la verdad de una manera, digamos, griega: como $\lambda \eta \dot{\theta} \theta \varepsilon \imath \alpha$ ó Aletheia, las cosas manifiestas, que no están ocultas. La búsqueda de la verdad estriba en 


\section{Una discusión en torno a la verdad en ciencias y humanidades}

descubrir la patencia de las cosas, apostando a que las cosas se nos revelen tal y como son. En su doble tradición, idealista y materialista, platónica y aristotélica, la ciencia ha querido buscar la verdad bajo la convicción de poder comunicarla racionalmente. Si el diálogo racional ayuda a mostrar la patencia de las cosas, la verdad de las cosas, racionalmente deberíamos poder justificar nuestras creencias sobre ellas. Así, históricamente, las formas convencionales de las teorías de la verdad fueron, al menos, de dos tipos: analítica y experimental. En las perspectivas analíticas, la verdad constituye un problema de lenguajes. En las perspectivas experimentales, un problema de confiabilidad, donde el foco es la factibilidad de realizar ciertas maniobras empíricas - naturales o de laboratorio- de modo replicable. En ambos casos, convencer de que las cosas son un asunto sobre lo evidente, en el dominio del razonamiento y la experiencia sensible.

Finalmente, la ciencia ha buscado la verdad de modo tan apasionado, que hoy la variedad y complejidad de las concepciones sobre la verdad son difíciles de resumir. La verdad de las teorías ha dado paso a las teorías de la verdad. Introduzcámonos a esta discusión mediante el problema de la aceptación de teorías científicas.

\section{VERDAD Y ACEPTACIÓN DE TEORÍAS}

Somos creyentes de que nuestras mejores teorías científicas son verdaderas, pues describen eficazmente el mundo. Pero la revisión histórica, como dijimos, nos alerta sobre esa falsa creencia. Entonces, ¿por qué seguir creyendo en las teorías científicas de hoy, si tenemos la expectativa que ellas se modifiquen en el futuro, incluso sustancialmente?

Nuestra óptica es que el problema de la verdad forma parte del problema más general de aceptación de teorías. Uno acepta una teoría porque supone que es verdadera y la rechaza porque supone que es falsa. Es decir, una teoría aspira a ser un retrato fidedigno del mundo, luego, verdadero del mundo. No tiene sentido aceptar una teoría que no dice la verdad. Sin embargo, la ciencia contemporánea ha consensuado que para aceptar una teoría, al menos, ésta debería aproximarse a la verdad o ser aproximadamente verdadera. Tal corrección de criterio ha servido para superar el impasse del problema de la inducción, donde según los más dogmáticos, un solo ejemplo en contra bastaría para descartar una teoría. También, ha servido para darle un propósito menos fotográfico y más relevante a la actividad científica: esto es, una actividad orientada a descubrir el modo de ser de las cosas, en vez de dedicarse a meras descripciones contingentes.

Las virtudes teóricas o epistémicas cognoscitivas, como su adecuación empírica, confirmación experimental, capacidad explicativo y predicciones 


\section{Jorge Gibert Galassi}

exitosas, no siempre han servido para caracterizar la aceptación de una teoría científica. En muchas ocasiones, las virtudes no cognoscitivas han sido esgrimidas para explicar la aceptación de una teoría, como los recursos retóricos (K. Knorr-Cetina), su compatibilidad con las formaciones discursivas o sistemas de positividad (Foucault) o paradigmas dominantes (Kuhn). Los factores sociales e históricos influencian la aceptación de una teoría y, por tanto, la verdad postulada por ellas. Tales factores juegan un rol, aunque sólo sea el de distorsionar involuntariamente la comprensión del mundo por parte de los "demasiado humanos" científicos.

La relación de la aceptación de teorías con la verdad de éstas dista de ser biunívoca. Pareciera que esto último sólo se da en el caso de las teorías más primitivas, que habitualmente se concentran en las entidades directamente observables. En la versión más simple, si la "T a" explica A directamente, entonces aceptamos "T a" como verdad de A. Usualmente el realismo científico ha trabajado con lo que se llama teoría de la verdad como correspondencia: la teoría se corresponde con los hechos.

Pero, ¿qué sucede con las teorías que postulan entidades inobservables? La distinción acá es entre realistas de teorías y realistas de entidades, o ambos (Dutra, 2003). Si una teoría habla sobre entidades inobservables, para un realista, quien acepta la teoría acepta la existencia de tales entidades, esto es, que ellas son reales. Si hay buenas razones epistémicas para aceptar una teoría, entonces hay buenas razones para aceptar como reales las entidades inobservables postuladas por ella (Sellars, 97). Pero el realismo de las teorías no necesariamente debe ir acompañado de un realismo de entidades. Puede darse el caso de que aceptamos la existencia de entidades inobservables pero no aceptamos las teorías correspondientes. Tal problema dice relación con la aceptación de las representaciones de las entidades. Ian Hacking (1983) postula que puede aceptar la existencia de un quark, ya que el trabajo experimental con los aceleradores de partículas permite manipular las partículas subatómicas, pero no se sigue que deba aceptar una teoría descriptiva de las entidades llamadas quark como verdadera, pues no disponemos de una buena descripción de ellas. Al revés, según Hacking, hay posturas que aceptan las teorías como reales pero no las entidades, como el planteamiento nominalista de Bertrand Russell, quien aceptaba que las teorías fuesen verdaderas, pero no que existieran las entidades inobservables que postulaba. Para Russell, términos como quark eran "resúmenes observacionales" y no correspondía a una entidad específica. Por ello, el realismo y la verdad de una teoría no implicaba una interpretación literal del lenguaje científico, sino lo contrario. Sin embargo, una interpretación literal del lenguaje científico puede sustentar, también, posturas anti-realistas, como

el realismo constructivo de van Fraseen. Para éste, un término como electrón 


\section{Una discusión en torno a la verdad en ciencias y humanidades}

sólo denota un objeto específico diferente de otros, como protón. Pero tales objetos no son reales, sino ficciones o meras representaciones: la ciencia no es sino una imagen, una representación nominal del mundo. No nos dice nada sobre su verdad y tampoco si pueden ser reales o no.

De tal manera que, según lo ya dicho, la aceptación de teorías científicas no está ineludiblemente ligada a la creencia en la verdad de ellas, así como, tampoco, en las entidades inobservables que postulan.

Afortunadamente, en esta diáspora, encontramos a Richard Boyd (1976, 1990), quien argumenta a favor de nuestra creencia intuitiva y "popular" sobre una ciencia que desarrolla teorías que son aceptadas por ser verdaderas respecto a las entidades que postula. Luiz Henrique de A. Dutra resume la discusión diciendo que algunos antirrealistas de las teorías, denominados instrumentalistas, pueden aceptar la virtud epistémica de ellas, cuando su verdad o valor de verdad es dejada en suspenso pero son consideradas buenas herramientas de predicción (como la física para Stephen Hawking) ${ }^{2}$ mientras que algunos antirrealistas de las entidades, como B. Russell, pueden aceptar el valor de verdad de una teoría. La discusión sobre la verdad de las teorías, según Dutra, se centra en la polémica entre realistas (de las teorías) e instrumentalistas; mientras que el problema de la existencia de entidades inobservables concentra la oposición entre realistas (de entidades) y nominalistas. Sobra decir que las teorías de la ciencia contemporánea hablan casi exclusivamente de entidades y propiedades inobservables (desde la noción de gravedad hasta la de quark), ya que ellas permiten explicaciones más ricas. Y no sólo en las ciencias naturales sino, también, en las ciencias sociales (integración social o capital simbólico).

\section{LA CONCEPCIÓN CLÁSICA: CONSECUENCIAS PARA LA DISCUSIÓN}

Las respuestas modernas a la búsqueda del conocimiento verdadero fueron muy influenciadas por Immanuel Kant, quien introdujo la distinción entre conocimientos a priori y a posteriori, según su relación con la experiencia como fuente, clasificando las proposiciones como analíticas o sintéticas. El rasgo característico de las primeras, según Kant, es su necesidad y universalidad, en contraste con la contingencia y particularidad de las proposiciones a posteriori.

En consonancia con ello, la llamada tercera antinomia kantiana - que opone el determinismo a la libertad - trajo como consecuencia la distinción entre el mundo natural y el mundo humano. La conexión kantiana entre mundo natural y determinismo promovió la masificación de la creencia en la

\footnotetext{
${ }^{2}$ Agradezco a Luiz H. de A. Dutra la aclaración sobre el instrumentalismo de S. Hawking.
} 


\section{Jorge Gibert Galassi}

ciencia como práctica orientada exclusivamente a la búsqueda de leyes universales (necesarias) y la confusión respecto del carácter único del determinismo como principio de causalidad, desde el siglo XVIII y casi hasta la mitad del siglo XX. El arraigo de esta creencia generó la plausibilidad de un distingo entre las ciencias naturales y las ciencias sociales, estas últimas idiográficas o "del espíritu", es decir, no susceptibles de asimilar concepto alguno de regularidad o patrón estable al estudiar los fenómenos históricos o sociales (de suyo, contingentes).

Pero la distinción analítico/sintético fue duramente cuestionada, pues supone un problema respecto de la condición de las leyes empíricas de la naturaleza que, según la misma propuesta kantiana, no son necesarias, pero deberían ser universales. En efecto, experimentalmente, la física cuántica barrió con los supuestos a priori de la ciencia natural kantiana, y W. O. Quine (1951) en su famoso artículo "Two Dogmas of Empiricism" criticó el apego del empirismo lógico a la doble identificación entre analítico-a priori y sintético-a posteriori y demostró que las teorías científicas no pueden ser puramente empíricas, pues están subdeterminadas (o infradeterminadas) por la teoría, lo que implica que cualquier teoría puede ser mantenida frente a cualquier evidencia observacional en el entendido que realicemos ciertos ajustes requeridos en nuestras creencias. Norwood Hanson (1958) desarrolló esta idea más conocida como la tesis del sesgo teórico de la observación (que desafortunadamente, se enseña poco en ciencias naturales; mientras que se enseña demasiado en ciencias sociales). Otro ataque provino desde la misma lógica, cuando Lewis y Langford escribieron Symbolic Logic (1932) y popularizaron el estudio de los razonamientos basados en las situaciones caracterizadas a través de las expresiones "necesariamente" y "posiblemente", además de las negaciones de ellas, como "contingentemente" e "imposible", llamados operadores modales. Finalmente, Saul Kripke (1980) sostuvo que hay proposiciones a priori que son contingentes y otras a posteriori que son necesarias.

En resumen, como no es posible decir que las ciencias y las humanidades responden a un patrón doctrinal exclusivo acerca de la verdad, trataremos de hacer un contrapunto entre las ciencias naturales y las ciencias sociales y humanidades.

\section{¿HACIA UNA DOCTRINA DE VERDAD —ADECUADA— PARA CADA DISCIPLINA?}

Si revisamos someramente, las teorías de verdad, la respuesta no es simple. Hay a lo menos tres tópicos vinculados: teorías de la verdad, teorías epistémicas de la verdad y teorías de la justificación. 
Una discusión en torno a la verdad en ciencias y humanidades

Elaboramos el siguiente cuadro a propósito del panorama sobre las teorías $(\mathrm{T})$ de la verdad

CUADRO No. 1: Teorías de la verdad

\begin{tabular}{|l|l|l|}
\hline \multicolumn{1}{|c|}{ T SUSTANTIVAS } & \multicolumn{1}{c|}{ T DEFLACIONARIAS } & \multicolumn{1}{c|}{ T FORMALES } \\
\hline T de correspondencia & T minimalista & T semántica \\
\hline T de la coherencia & T de la denotación & T de la prueba \\
\hline T constructivista & T performativa & T de los modelos \\
\hline T del consenso & T de la redundancia & T de Kripke \\
\hline T pragmática & & \\
\hline
\end{tabular}

Las teorías sustantivas tratan la verdad como algo natural, fenoménico o experiencial sobre lo cual puede ser dicho algo significativo. La teoría de la correspondencia es, lejos, la teoría más aceptada y plantea que las creencias y afirmaciones verdaderas se corresponden con el estado actual de los hechos. Vincula de manera estricta nuestros pensamientos o estados mentales con las cosas $\mathrm{u}$ objetos externos. La verdad es la copia del mundo en nuestros pensamientos y supone que hay una realidad objetiva que se refleja en nuestra capacidad cognoscitiva. Las objeciones a esa postura nacen de la consideración elemental que tal reflejo debe ser trascripto a algún lenguaje, donde nacen problemas de traducción que ameritan una teoría formal (semántica). La teoría de la correspondencia funciona igual de eficaz tanto en ciencias naturales como sociales. La teoría de la coherencia, en tanto, se funda en la idea de que la verdad es básicamente una propiedad de un sistema total de proposiciones; luego las proposiciones individuales cuentan sólo en concordancia con su coherencia respecto del sistema total. A diferencia de la primera, esta teoría ha sido criticada por su falta de justificación para ser aplicada a las proposiciones del mundo empírico, en general, especialmente la psicología y las ciencias sociales, en virtud de la constatación elemental de nuestra incoherente conducta cotidiana. Por otro lado, el hecho primordial de que todas las teorías sociales aceptan que la sociedad es construida históricamente, ha dado pie a lo que se conoce como las teorías constructivistas de la verdad. Estas teorías sugieren que la verdad es construida por procesos sociales, culturales y por las luchas de poder dentro de las comunidades. La famosa frase que dice "la historia la escriben los vencedores" sugiere que es difícil distinguir la verdad de lo que pasó con la "historia oficial". Una variante de lo anterior es la teoría de la verdad como consenso, donde el contenido puede ser cualquier cosa siempre y cuando un grupo o comunidad esté de acuerdo en ello. La implicancia de ambas teorías, constructivista y del consenso, es lejos la que ha remecido la epistemología de la ciencia y de las humanidades en los últimos cuarenta años, pues finalmente ¿quién si no los científicos — como comunidad en guerra o de acuerdo- 


\section{Jorge Gibert Galassi}

postulan qué teorías científicas son aceptadas como verdaderas y cuáles son rechazadas como falsas? Finalmente, una muy atractiva teoría de la verdad es la pragmática, cuya influencia en las ciencias aplicadas y tecnológicas tiene razones de Perogrullo. Según ésta, la verdad es confirmada por el resultado positivo tras haber puesto la proposición en práctica.

Las teorías deflacionarias de la verdad se desarrollaron como respuesta a la insistencia en el uso de los predicados de verdad y al rechazo de que el concepto de verdad refiere a una propiedad real de una sentencia o proposición. Así, según esta escuela, " $2+2=4$ " "es verdad" posee equivalencia lógica con $2+2=4$, luego la frase "es verdad" está de sobra. En este marco, las teorías minimalistas y denotacionales son sólo otra expresión para las teorías deflacionarias, pues todas abogan por la supresión de notaciones especiales e innecesarias. La teoría performativa, por su parte, afirma que decir "la nieve es blanca, es verdad" constituye el desempeño de un acto de habla que señala el acuerdo respecto de la proposición "la nieve es blanca". Luego, la extensión "es verdad" cumple un rol importante en esta teoría. Cuando un presidente electo dice en la ceremonia de ascensión a la primera función de gobierno: "Sí, juro como presidente" no sólo es una descripción sobre sí mismo, sino un acuerdo que implica asumir las responsabilidades y derechos del cargo de inmediato. Muchas proposiciones son más acciones que actos comunicativos. Las teorías de la redundancia concuerdan con la idea minimalista, pero infieren que la verdad es un concepto redundante. Las implicaciones para los lenguajes de las teorías científicas y las realidades hacia las cuales éstos apuntan son decisivos y rayan en la discusión ontológica. Por ejemplo, decir "la integración social es fuerte en la sociedad $Y$ " podría ser deflacionado diciendo "Y es una sociedad con integración social", pues, quizás, no hay grados de integración social; quizás, no hay integración social débil y, quizás, si no está integrada, tal sociedad y está fragmentada.

Finalmente, están las teorías formales, entre las que destacan las teorías de la verdad matemática. La teoría de modelos es el estudio de las estructuras matemáticas a través de fórmulas lógicas y la teoría probatoria, por su parte, centra su interés en la demostración, y es una rama de la lógica matemática que provee demostraciones de objetos matemáticos formales. Quizás la más famosa teoría sea la de Alfred Tarski, quien desarrolló la teoría semántica de la verdad para lenguajes formales. En términos breves, la teoría de Tarski dice que ningún lenguaje puede contener su propio predicado de verdad, es decir, que la expresión 'es verdad' sólo puede ser aplicada a proposiciones en algún otro lenguaje distinto al de ellas, para evitar las proposiciones formalmente paradójicas como 'estoy mintiendo'. Ahora bien, generalmente en ciencias, la formalización de teorías ha sido una aspiración distintiva. El estudio estático 
de las teorías científicas se refiere precisamente a la forma o estructura lógica de las teorías y se centra básica, pero no exclusivamente, en el carácter deductivo (o deducibilidad) de los enunciados particulares desde los generales. La relación de deducibilidad entre fórmulas en las ciencias empíricas permiten la generación de sistemas de hipótesis, que se controlan - o pueden hacerlo - unas a otras (Bunge, 1969). Según Bunge, es el caso de la mecánica de Newton, la teoría de la evolución de Darwin y la teoría conductista de Hull. En el caso de las ciencias sociales, por su naturaleza (o por desidia histórica), no ha sido posible su fundamentación mediante procedimientos formales, aun cuando algunas teorías macroeconómicas se han formulado de acuerdo con axiomas abstractos como el comportamiento racional de los agentes económicos. De algún modo, ha persistido la creencia que no hay una teoría formal suficientemente adecuada para reducir la riqueza y heterogeneidad de la vida social, obviando el hecho de que lo que se debe formalizar son las teorías, pues el "mundo" es irreductible. El lenguaje de la probabilidad, por mencionar una formalización trivial, está permanentemente actualizando nuestra comprensión de un amplio rango de fenómenos sociales. La sociología matemática, por ejemplo, ha permitido descubrir y comprender mejor muchas estructuras subyacentes en la realidad social, inclusive, bastante contrarias al sentido común, como el hecho que en muchos países los índices de felicidad no tienen correlación alguna con la calidad de vida en ellos. Finalmente, dentro de las teorías formales, está la teoría de la verdad de Kripke, que surge de la demostración que un lenguaje natural es capaz de contener predicados de verdad sin caer en contradicción, y que hay proposiciones que no son verdaderas ni falsas, que contradicen el principio de bivalencia. Este tipo de lógica comenzó en 1959 cuando el estudiante de Harvard (de 19 años) Saul Kripke introduce la lógica ahora conocida como semántica modal. El término lógica modal cubre una vasta familia de lógicas con reglas similares, entre ellas, la lógica deóntica ("es obligatorio que..."), la temporal ("fue el caso que...") y la doxástica o de la opinión ("Juan cree que...").

Pero mientras las teorías precedentes tratan la verdad como algo sobre lo cual puede ser dicho algo significativo o correcto, las teorías epistémicas de la verdad apuntan más bien a las implicaciones gnoseológicas como el problema del conocimiento, el rol de las creencias, la aceptación o refutación de proposiciones y los criterios de perspectiva.

CUADRO No. 2: Teorías epistémicas de la verdad (TE)

\begin{tabular}{|l|l|l|}
\hline \multicolumn{1}{|c|}{$\begin{array}{c}\text { TEORÍAS EPISTÉMICAS } \\
\text { VERIFICACIONISTAS }\end{array}$} & $\begin{array}{c}\text { TEORÍAS EPISTÉMICAS } \\
\text { PERSPECTIVISTAS }\end{array}$ & $\begin{array}{c}\text { TEORÍAS EPISTÉMICAS } \\
\text { PRAGMÁTICAS }\end{array}$ \\
\hline positivismo & individual & consenso racional \\
\hline apriorismo & discursive & \\
\hline falsacionismo (Popper) & colectivo & \\
\hline & trascendental & \\
\hline
\end{tabular}




\section{Jorge Gibert Galassi}

Hemos agrupado bajo el calificativo de verificacionistas, todas aquellas doctrinas que se definen desde el problema de qué hacer con la evidencia. En general, la historia de la ciencia ha sido verificacionista, pues se ha centrado en verificar o comprobar hipótesis. El resultado de tales comprobaciones ha sido tildado de "verdadero", con lo que la verdad ha sido reducida a la verificación. Esta concepción está muy internalizada en nuestra vida diaria. Uno dice: "no hay que dudar más, está comprobado". El empirismo lógico del círculo de Viena asumió la distinción kantiana entre verdades de hecho y verdades lógicas; luego generó dos alternativas: el positivismo y el apriorismo. El primero postula que una proposición es significativa, o capaz de ser verdadera o falsa, si y sólo si ésta es verificable por la experiencia sensible. El apriorismo, por el contrario, se apoya en la razón y en su capacidad de sostener una proposición mediante un razonamiento que la verifica a priori, intelectualmente, con certeza. El positivismo lógico (no mencionado en el cuadro) trata de combinar ambas doctrinas. Pero, ¿qué es una experiencia sensorial significativa o indicativa de verdad? Desde la invención del espectroscopio, sabemos que nuestra representación homogénea de la luz es errónea. Ya en el segundo apartado hemos discutido varios puntos críticos de estas concepciones, como el instrumentalismo y el nominalismo. La idea de Popper sobre la verdad es distinta. Debemos resignarnos a las verdades 'hasta nuevo aviso' y la manera de lograrlo es buscar evidencias y argumentos que destruyan nuestras hipótesis. Así, mediante una suerte de sobrevivencia del más apto, las ideas que sean capaces de resistir tal embate pueden ser consideradas verdaderas, hasta que ellas sean abatidas $\mathrm{y}$ reemplazadas por otras ideas: más próximas a la verdad o verosímiles. A pesar de que el programa de Popper terminó abruptamente con la refutación de Tichy $(1974,1976)$ — aceptada por Popper en 1978 - habría que señalar que su doctrina antiinductivista hipotética elevó la prudencia y el rigor de las prácticas confirmacionistas.

El segundo grupo de teorías epistémicas son las perspectivistas que, brevemente, se sustentan en la afirmación que la verdad sólo es relativa a una perspectiva particular. Esta postura también ha sido muy internalizada en nuestra convivencia diaria: "nada es verdad, nada es mentira, todo depende del cristal con que se mira". Se reconocen cuatro versiones de esta noción. El perspectivismo individual afirma que una proposición es verdadera para una persona si y sólo si es aceptada o creída por esa persona. En las humanidades, siempre ha sido así. Es la verdad "de autor", la vida según Séneca o la sabiduría según Buda. Desafortunadamente, esta tendencia ha sido exportada a las ciencias sociales, cuyos cultores bogan por la teoría de Anthony Giddens o por las de Pierre Bourdieu, en lugar de centrarse en los objetos empíricos o marcos teóricos comunes. El perspectivismo discursivo, también denominado 


\section{Una discusión en torno a la verdad en ciencias y humanidades}

convencionalismo, postula que una proposición es verdadera en relación a un sistema de discurso (escogido de manera convencional, según preferencia estética, política o moral) si y sólo si consiste en una afirmación producida o legitimada por los métodos de ese particular discurso. El mejor ejemplo de esto es el formalismo, doctrina matemática que postula que la verdad de una afirmación es relativa a un conjunto de supuestos, siempre que tal afirmación sea consecuencia deductiva de tales supuestos. Como vimos en las teorías de la verdad formal, en general las ciencias buscan la coherencia entre sus enunciados, por lo que cada teoría es un sistema conceptual. Ahora bien, la diferencia entre el perspectivismo discursivo y una teoría formal cualquiera es que los lenguajes son muy distintos. El perspectivismo discursivo ha sido fatal para las ciencias fácticas, sean éstas naturales o sociales. No pocas veces ha servido de excusa para una interpretación dogmática de la realidad, y como todo buen discurso, llega a veces a confundirse con la realidad o la reemplaza, producto de sus recursos retóricos obscuros, con aureola de misterioso 'absoluto' (sólo disponible para unos pocos elegidos). Sin embargo, el perspectivismo discursivo ha tenido el mérito de alertarnos respecto del hecho crucial de que nos comunicamos y coordinamos como especie mediante lenguajes, donde muchos de los sistemas —en especial los semánticosoperan en base a creencias implícitas. Las ideologías políticas son buenos ejemplos. Siendo discursos que en sus orígenes estaban de algún modo anclados en los hechos, posteriormente los empiezan a derivar de las creencias primitivas de su sistema de proposiciones y métodos discursivos. En ciencias sociales, otro fundamento epistémico importante son las teorías de la verdad en sociología de la ciencia, usualmente etiquetadas como perspectivismo colectivista. La interpretación en estas teorías centra su atención en que la verdad es construida por grupos sociales. La verdad se entiende referida a tradiciones y culturas (incluidas la tradición científica) que dan por descontado que su propia visión de las cosas entraña la verdad de ellas. Así, una proposición es verdadera para una comunidad si y sólo si hay un consenso entre los miembros de la comunidad para creerla. Es la versión 'consensual' del perspectivismo colectivo. La comunidad científica, por ejemplo, según Robert K. Merton (1977), se caracteriza por un conjunto de valores comunes, normas y prácticas; de lo que resultan creencias compartidas. La otra versión es aquella que indica que la verdad es poder, luego, basta administrar poder para sacramentar verdades. En esta dimensión, una perspectiva colectiva nace de una comunidad fortalecida por privilegios de poder. La Iglesia, en la Edad Media, era una de tales comunidades; mientras que en la época moderna lo son los médicos, entre otros grupos. El tercer caso del perspectivismo colectivo es el de la tradición marxista, que enfatiza la generación de la verdad mediante la lucha contra el poder burgués, 


\section{Jorge Gibert Galassi}

generación que es producto de la emancipación del proletariado, que devela el carácter alienado de la organización capitalista de la sociedad. Otro caso de perspectivismo colectivo es el de Pierre Bourdieu. Su teoría del campo científico sustenta un espacio social que construye la verdad en una lucha que los innovadores ganan, sólo si conservan los capitales simbólicos que reglan la dinámica del campo, en una suerte de transición lenta de una verdad a otra según procedimientos regulados, y que se integran progresivamente gracias a la confrontación racional. La postura de Bourdieu no niega el progreso científico, pero, al mismo tiempo, afirma que "la verdad es la relatividad generalizada de los puntos de vista (de los agentes científicos: individuos, grupos e instituciones) al interior del campo" (2003:198). Además de ellas, está el perspectivismo trascendental que implica una perspectiva ideal valedera, fuera del alcance humano inmediato.

El tercer grupo es el de las teorías pragmáticas que, a menudo, son combinaciones de todas las anteriores. Desde esa óptica, no es estrictamente clasificable como una teoría distintiva, aunque tiene puntos de contacto con las teorías de la verdad y de la investigación científica en tanto adquisición de conocimientos. Entre ellas, una postura relevante es la del consenso modificado que debe satisfacer ciertas restricciones exógenas a las preferencias epistémicas arbitrarias de los miembros del grupo que lo adopta, como la norma de criticidad y de reproducibilidad de los métodos. La "Teoría de la acción comunicativa" de Jürgen Habermas (1985) ha sido vista, por muchos, como basada en tal concepto epistémico de verdad.

Las agrupaciones precedentes (Teorías de la verdad y Teorías epistémicas de la verdad) pueden ser vistas como soportadas o edificadas en base a determinados esquemas de justificación de las creencias que las hacen plausibles. El cuadro 3 resume esta visión.

CUADRO 3: Teorías de la justificación

\begin{tabular}{|l|l|}
\hline \multicolumn{1}{|c|}{ FUENTES DE JUSTIFICACIÓN } & \multicolumn{1}{c|}{ ELEMENTOS PRIMITIVOS } \\
\hline internalismo & coherentismo \\
\hline externalismo & fundacionalismo \\
\hline & fundherentismo \\
\hline
\end{tabular}

Las teorías de la justificación pueden ser clasificadas desde un criterio de origen o de articulación de elementos primarios. Desde las fuentes, el internalismo justifica las creencias desde un tipo de conocimiento interno, que puede ser a priori, racional o meramente revelado. Desde el externalismo, las fuentes externas de conocimiento pueden ser usadas para justificar una creencia, por ejemplo, un indicador empírico o, bien, una voz autorizada. Por otro lado, y éste es el prisma más fructífero, los elementos se articulan por su razonabilidad o su coherencia. Así, el fundacionalismo dice que "algunas 


\section{Una discusión en torno a la verdad en ciencias y humanidades}

creencias justificadas son básicas (empíricas o no empíricas); una creencia básica está justificada independientemente del apoyo de cualquier otra creencia" (Haack 30); mientras que el coherentismo indica que "una creencia está justificada si pertenece a un conjunto coherente de creencias" (34). Pero, Haack rechaza ambas perspectivas, no por erróneas sino por insuficientes. Postula el fundherentismo que caracteriza como una doctrina donde "la experiencia del sujeto es importante para la justificación de sus creencias empíricas, pero no es necesario que exista una clase privilegiada de creencias empíricas justificadas exclusivamente por el apoyo de la experiencia, independientemente del apoyo de otras creencias" (37).

Como vemos, la riqueza de la discusión es notable. Veamos cómo podemos aportar para orientar los estudios futuros sobre el tema.

G. K. Chesterton afirmó en 1905 "solamente puede encontrar la verdad con la lógica si usted ya encontró la verdad sin ella". Desde esa aproximación, bastaría la mera información sobre algo (como la afirmación "usted está leyendo este texto") para intuir la verdad de ello. Pero, la frase puede llevar a engaño, pues, lógica y verdad pueden ser excluyentes, dado que un razonamiento puede ser válido aunque usemos proposiciones falsas. Además, como hemos visto, muchas teorías de la verdad son formales y otras más se apoyan en las afirmaciones empíricas. El asunto es, más bien, que en el contexto de nuestro trabajo, las teorías científicas - sin los apellidos de "natural" o "social" - si son representadas lógicamente y contienen al menos si un axioma con contenido empírico contrastable, pueden ser verdaderas en algún sentido gnoseológico. Es decir, pueden constituir conocimiento.

En las ciencias modernas, especialmente en la mecánica cuántica, esto no es fácil y ha dado pie a la postura de la verdad teórica como imagen científica o representación. La pluralidad de representaciones sobre un objeto ciertamente avala el perspectivismo científico, pero no el constructivismo, aun cuando la tendencia actual es a estrechar la distancia entre ambas doctrinas (Giere, 2006). La ciencia dice algo 'objetivo' sobre la realidad, pero no de una manera única. El hecho de que filósofos como David Stove hayan calificado la sociología de la ciencia como algo estúpido (2006), se debe al efecto Ismael, que resumidamente indica que sólo los sociólogos de la ciencia - como Ismael, único sobreviviente en la novela Moby Dick- se autoatribuyen la capacidad de superar los determinismos histórico-sociales y epistémicos que ellos postulan como imposibles de superar. Por fortuna, las fuerzas de la naturaleza son 'ciegas' y, por tanto, no existe intención alguna de parte del mundo natural de burlar nuestra comprensión de sus procesos y estructuras. 


\title{
Jorge Gibert Galassi
}

\section{CONCLUSIONES PRELIMINARES}

En síntesis, en las ciencias sociales y en las humanidades, siempre existe el fantasma del voluntarismo (en buena hora). Es decir, sospechamos que las cualidades o propiedades del mundo social son distintas a las del mundo natural. En especial, hay buenos argumentos respecto a que las estructuras, procesos y actos sociales no responden, en general, a una lógica darwiniana o mecánica. La verdad en el mundo social no sólo debe lidiar con el perspectivismo sino que, también, con el libre albedrío. Jorge Luis Borges en "La muerte y la brújula", relató "Es verdad que Erik Lönnrot no logró impedir el último crimen, pero es indiscutible que lo previó" (1944). Es decir, las descripciones pueden ser verdaderas y no verificarse. Y cabe, también, la posibilidad contraria; una descripción falsa puede generar autoverificaciones: "si un grupo define una situación como real, las consecuencias de ello son reales". Tal posibilidad, formulada por William I. Thomas en 1928 y conocida como el teorema de Thomas - desarrollada por Merton a partir del concepto de Profecía autocumplida (1995) - remite al problema de la verdad fáctica generada a partir de irrealidades. En situaciones de la vida social no hay falacia de afirmación del consecuente.

En ese sentido, la distinción real entre la verdad del mundo social y la del mundo natural es simple: en el primer caso, la verdad de una teoría está mediada por la aceptación que el objeto hace de ella y, en el segundo caso no lo está, pues ningún gen se tomará la molestia en desacreditar su descripción por ella. Mientras las teorías naturales deben someterse, sólo en primera instancia, a la crítica — de la comunidad científica-, las teorías sociales y las humanidades deben hacerlo dos veces: primero, ante la comunidad científica y, luego, ante la comunidad, en general, su objeto transformado en sujeto.

\author{
Universidad de Viña del Mar* \\ Campus Rodelillo \\ Av. Agua Santa 7255 \\ Viña del Mar (Chile) \\ jgibert@uvm.cl
}


Una discusión en torno a la verdad en ciencias y humanidades

\section{BIBLIOGRAFÍA}

BOURDIEU, Pierre. El oficio de científico. Barcelona: Anagrama, (2003).

BOYD, Richard. "Realism, approximate truth and philosophical method", in Savage (org.). Scientific theories. Minnesota Studies in Philosophy of Science, vol. XIV. Minneapolis: University of Minneapolis Press, 1990, 355-91.

(1976): “Approximate truth and natural necessity", en Journal of philosophy 73: 633-5.

BUNGE, Mario. La investigación científica. Barcelona: Ariel, 1969.

DUTRA, Luiz Henrique. Introducao a teoria da ciencia. Florianópolis: Editora da UFSC, 2003.

GIERE, Ronald. Scientific perspectivism. Chicago: University of Chicago Press, 2006.

HAACK, Susan. Evidencia e investigación. Madrid: Tecnos, 1997.

HABERMAS, J. (1985). Teoría de la acción comunicativa. Madrid: Taurus, 1987.

HACKING, Ian. Representing and intervening. Cambridge: Cam-bridge University Press, 1983.

HANSON, Norwood. Patterns of scientific discovery. Cambridge: CUP, 1958.

KRIPKE, Saul. Naming and necessity. Cambridge: Harvard University Press, 1980.

KUHN, Thomas. The trouble with the historical philosophy of science. Harvard University (Department of the History of Science), Cambridge, MA, 1992.

LEWIS, Clarence y Langford, Cooper (1932). Symbolic logic. NYC: Dover publications, 2000.

MERTON, R. La sociología de la ciencia. Madrid: Alianza, 1977.

------- The Thomas Theorem and the Matthew effect. Social Forces 74 (2): (1995):379-424.

MICHAEL, Foucault. Estrategias de poder. Barcelona: Paidós, 1999.

THOMAS, W.I. The child in America: Behavior problems and programs. NYC: Knopf, 1928.

TICHY, P. "On Popper's Definitions of Verisimilitude", The British Journal for the Philosophy of Science 25, (1974):155-160.

------ "Verisimilitude redefined", The British Journal for the Philosophy of Science 27, (1976): 25-42.

STOVE, David. The Plato cult: and other philosophical follies. Oxford: Blackwell, 1991. 


\section{Jorge Gibert Galassi}

SELLARS, W. Science, perception and reality. Londres: Routledge \& Keagan Paul, 1963.

VAN Fraassen, Bas. The scientific image. Oxford: Clarendon Press, 1980. 